\title{
44 Tau: Discrimination between MS and post-MS models
}

\author{
P. Lenz, ${ }^{1}$ A. A. Pamyatnykh, ${ }^{1,2,3}$ M. Breger, ${ }^{1}$ V. Antoci ${ }^{1}$ \\ ${ }^{1}$ Institut für Astronomie, Türkenschanzstrasse 17, 1180 Vienna, Austria \\ ${ }^{2}$ Copernicus Astronomical Center, Bartycka 18, 00-716 Warsaw, Poland \\ ${ }^{3}$ Institute of Astronomy, Pyatnitskaya Str. 48, 109017 Moscow, Russia
}

\section{Observations and Mode Identification}

Antoci et al. (2006) analysed photometric data of 44 Tau from $2000-2003$ and detected 29 oscillation frequencies of which 13 are independent. We performed a mode identification based on the amplitude ratios and phase differences from the photometric data set of 2000/01. As shown by Daszyńska-Daszkiewicz et al. (2003), the results are very sensitive to the treatment of convection in the envelope. We find that in the case of 44 Tau only models with ineffective convection $\left(\alpha_{\text {conv }} \approx 0\right)$ result in definitive mode identifications. The observed modes $\mathrm{f} 1$ $(6.8980 \mathrm{c} / \mathrm{d})$ and $\mathrm{f5}(8.9606 \mathrm{c} / \mathrm{d})$ can definitely be identified as $\ell=0$ modes. Their frequency ratio 0.7698 is close to the typical ratio of the radial fundamental and first overtone frequencies in the $\delta$ Scuti domain. Four modes are identified as $\ell=1$ and two other modes as $\ell=2$ modes. Two $\ell=1$ modes $(9.1175$ and $9.5613 \mathrm{c} / \mathrm{d})$ and both $\ell=2$ modes look to be close to the avoided crossing stage and may be used as indicators of the efficiency of overshooting from the stellar convective core.

\section{Modelling 44 Tau}

From the HIPPARCOS parallax, Strömgren and Geneva photometry and from the Vienna grid of stellar atmospheres (Nendwich et al., 2004) we derive $\log L / L_{\odot}=1.34 \pm 0.07$ and $T_{\text {eff }}=$ $6900 \pm 100 \mathrm{~K}$. With a $\log g$ value of $3.6 \pm 0.1$ it is not possible to determine the evolutionary status of 44 Tau unambiguously.

Our main sequence models of 44 Tau that can fit the radial fundamental and first overtone generally are too cool and in some cases too faint. An acceptable fit of all identified modes can be obtained only for enhanced metal abundance and/or significant overshooting from the convective core.

In the post-MS case it is possible to obtain a model within the observational error box in the HR Diagram with no need of overshooting and nonstandard chemical composition. For post-MS models we predict much more unstable modes than we observe. A possible explanation why only specific modes are observed is mode trapping in the stellar envelope. The predicted frequency spectra for the MS and post-MS case are given in Fig. 1.

\section{Conclusions}

For both MS and post-MS models it is possible to obtain good fits to the observed frequency spectrum. However, the MS models are significantly cooler than it can be estimated from photometry. Considering the good fit of both the observed frequencies and physical parameters, standard post-main sequence models with inefficient convection seem to be preferable.

Acknowledgments. We would like to thank Rafa Garrido and Juan Carlos Suárez for valuable discussions during the conference. This work has been supported by the Austrian FWF. AAP acknowledges the financial support from HELAS and from the Polish MNil grant No. 1 P03D 02128. 


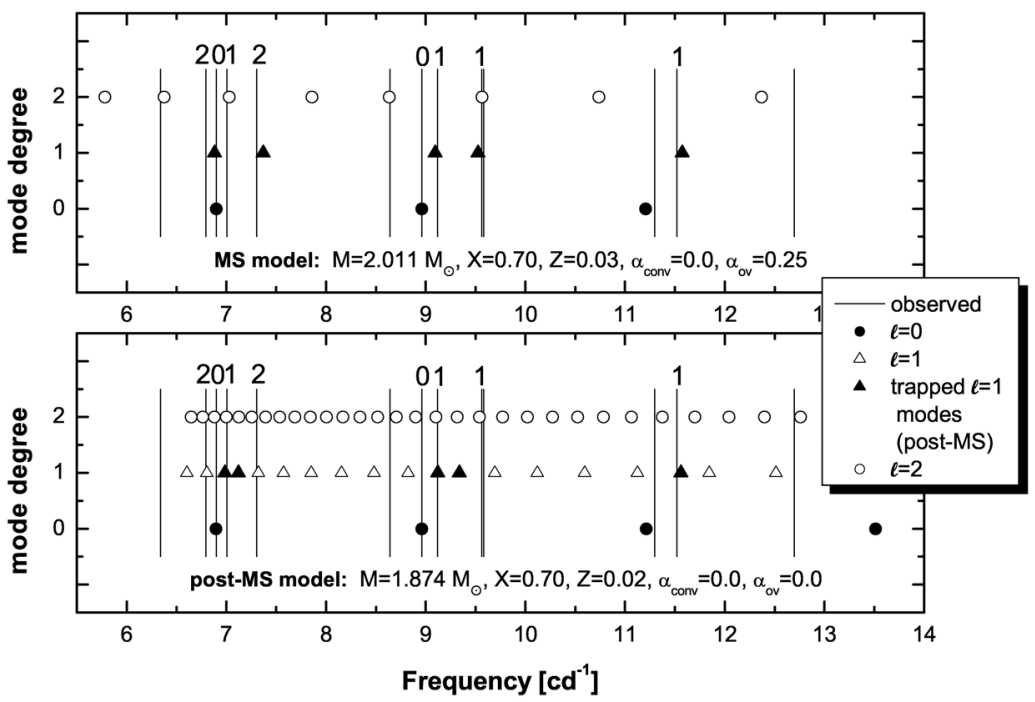

Figure 1: Comparison of the predicted frequency spectrum with observations for a selected main sequence model (upper panel) and a post-MS model (lower panel). In the post-MS case trapped $\ell=1$ modes are indicated by different symbols.

\section{References}

Antoci V., Breger M., Rodler F., Bischof K., Garrido R., 2007, A\&A, 463, 225

Daszyńska-Daszkiewicz J., Dziembowski W. A., Pamyatnykh A. A., 2003, A\&A 407, 999

Nendwich J., Heiter U., Kupka F., Nesvacil N., Weiss W. W., 2004, Comm. Asteroseis., 144, 43 